Arq. Bras. Med. Vet. Zootec., v.62, n.4, p.797-800, 2010

\title{
Aplasia segmentar de corpo uterino em cadela sem raça definida: relato de caso
}

[Segmental aplasia of the uterine body in a mongrel bitch: a case report]

\author{
M.V.D. Almeida ${ }^{1}$, E.P. Rezende ${ }^{1}$, A.R. Lamounier ${ }^{2}$, M.A. Rachid ${ }^{2}$, \\ E.F. Nascimento ${ }^{3}$, R.L. Santos ${ }^{3}$, G.R. Valle $^{2 *}$ \\ ${ }^{1}$ Aluno de graduação em Medicina Veterinária - PUC Minas - Betim, MG \\ ${ }^{2}$ Departamento de Medicina Veterinária - PUC Minas - Betim, MG \\ ${ }^{3}$ Escola de Veterinária - UFMG - Belo Horizonte, MG
}

\begin{abstract}
RESUMO
Relata-se um caso de aplasia segmentar parcial de corpo uterino em uma cadela sexualmente madura, em anestro, sem raça definida, em bom estado clínico geral. À necropsia, não foram identificadas alterações dos demais órgãos genitais, à exceção do corpo uterino, que apresentava redução de espessura numa extensão de $2 \mathrm{~mm}$ na parte mediocaudal, com ausência de lúmen nesse ponto, e hidrometra em sua porção cranial e cornos uterinos. A avaliação histológica evidenciou redução da espessura total do corpo uterino no seu terço mediocaudal, porém presentes perimétrio, miométrio e endométrio, mas ausência de lúmen, bem como redução da espessura total da parede uterina nos cornos, com redução do número de glândulas endometriais. Nos ovários, foram identificados folículos em vários estádios de desenvolvimento e corpos lúteos em regressão, indicando que a cadela estava em atividade cíclica normal.
\end{abstract}

Palavras-chave: cadela, aplasia segmentar, corpo uterino, hidrometra

\begin{abstract}
A case of partial segmental aplasia of the uterine body in a sexually mature mongrel bitch in anestrous phase and normal clinical condition is reported. At necropsy, no changes in the genital organs were observed with the exception of the uterine body that had a segmental ( $2 \mathrm{~mm}$ in length) decrease in diameter in its medial-caudal portion, with absence of lumen and hydrometra in the cranial portion of the uterine body and uterine horns. Histopathologic evaluation indicated reduction in thickness of the whole uterine body at the site of stenosis, with all layers preserved including perimetrium, myometrium, and endometrium, but with the absence of a lumen. The walls of the uterine horns were thin and there was a marked decrease in the number of endometrial glands. There were several follicles on different stages of development and corpora lutea in regression, indicating that the bitch had normal cyclic activity.
\end{abstract}

Keywords: bitch, segmental aplasia, uterine body, hydrometra

\section{INTRODUÇÃO}

A aplasia segmentar é uma anomalia do desenvolvimento do sistema genital tubular feminino, condição provavelmente hereditária que causa desenvolvimento incompleto dos ductos paramesonéfricos durante a fase embrionária (Johnston et al., 2001; Nascimento e Santos, 2003; Schlafer e Miller, 2007), os quais dão origem à porção cranial da vagina, cérvix, útero e tubas uterinas (Schlafer e Miller, 2007). É uma condição comum na vagina e no útero de vacas e porcas, sendo geralmente acompanhada de hidrometra ou mucometra nas porções craniais ao segmento aplásico, podendo haver anestro prolongado devido à persistência de corpo lúteo nessas espécies (Nascimento e Santos, 2003).

Recebido em 20 de agosto de 2009

Aceito em 16 de julho de 2010

*Autor para correspondência (corresponding author)

E-mail: guilhermerv@pucminas.br

Apoio: PROBIC - PucMinas/FAPEMIG 2665/2008 
Em cadelas, no entanto, é uma condição rara (Johnston et al., 2001; Nascimento e Santos, 2003), tendo sido encontrado apenas um caso em 300 cadelas de rua examinadas (Ortega-Pacheco et al., 2007). São descritos como aplasia segmentar vaginal (Wadsworth et al., 1978), útero unicorno (Ortega-Pacheco et al., 2007), aplasia total de corpo uterino (Oh et al., 2005), aplasia segmentar em um dos cornos (Prestes et al., 1997; Schulman e Bolton, 1997) e comprimento reduzido dos cornos uterinos (Johnston et al., 2001). Geralmente a genitália externa, as tubas uterinas e os ovários das cadelas afetadas estão normais (Johnston et al., 2001; Schlafer e Miller, 2007), assim como a atividade cíclica (Johnston et al., 2001), mas podem estar associados à hidrometra (Oh et al., 2005) e ao complexo hiperplasia endometrial cístico/piometra (Prestes et al., 1997; Schulman e Bolton, 1997).

\section{CASUÍSTICA}

Ao ser necropsiada após eutanásia, como parte do grupo-controle de um experimento, uma cadela sexualmente madura, sem raça definida, apresentava aplasia parcial de corpo uterino, numa extensão de $2 \mathrm{~mm}$, com acúmulo cranial de líquido intrauterino. Como parte das avaliações realizadas no referido experimento, estavam normais o hemograma - hematócrito, 40\%; contagem de hemácias, 5,97 x $10 \% / \mathrm{mm}^{3}$, plaquetas, $113,5 \times 10^{6} / \mathrm{mm}^{3}$ e total de leucócitos, $3,05 \times 10^{6} / \mathrm{mm}^{3}-$, os perfis bioquímicos plasmáticos para função hepática e renal proteínas totais, 3,6g/dL; albumina. $0,9 \mathrm{~g} / \%$; bilirrubinas direta, $0,4 \mathrm{mg} / \mathrm{dL}$, indireta, $0,1 \mathrm{mg} / \mathrm{dL}$ e total, $0,5 \mathrm{mg} / \mathrm{dL}$; gamaglutamil transferase, 1,9UI/L; transaminase glutâmico-oxalacética, 74UI/L; transaminase glutâmico-pirúvica, 58,5UI/L; creatinina, $0,5 \mathrm{mg} / \mathrm{dL}$; ureia, $24 \mathrm{mg} / \mathrm{dL}$ -, bem como a histologia hepática e renal. A citologia vaginal e a dosagem sérica de progesterona $(0,4 \mathrm{ng} / \mathrm{mL})$ mostraram que a cadela estava em anestro.

Macroscopicamente observou-se redução da espessura do corpo uterino em sua parte mediocaudal, estando a parte cranial do corpo e os cornos uterinos repletos de secreção transparente inodora, caracterizando hidrometra (Fig. 1). Vulva, vagina, cérvix, tubas uterinas e ovários apresentavam-se macroscopicamente normais.

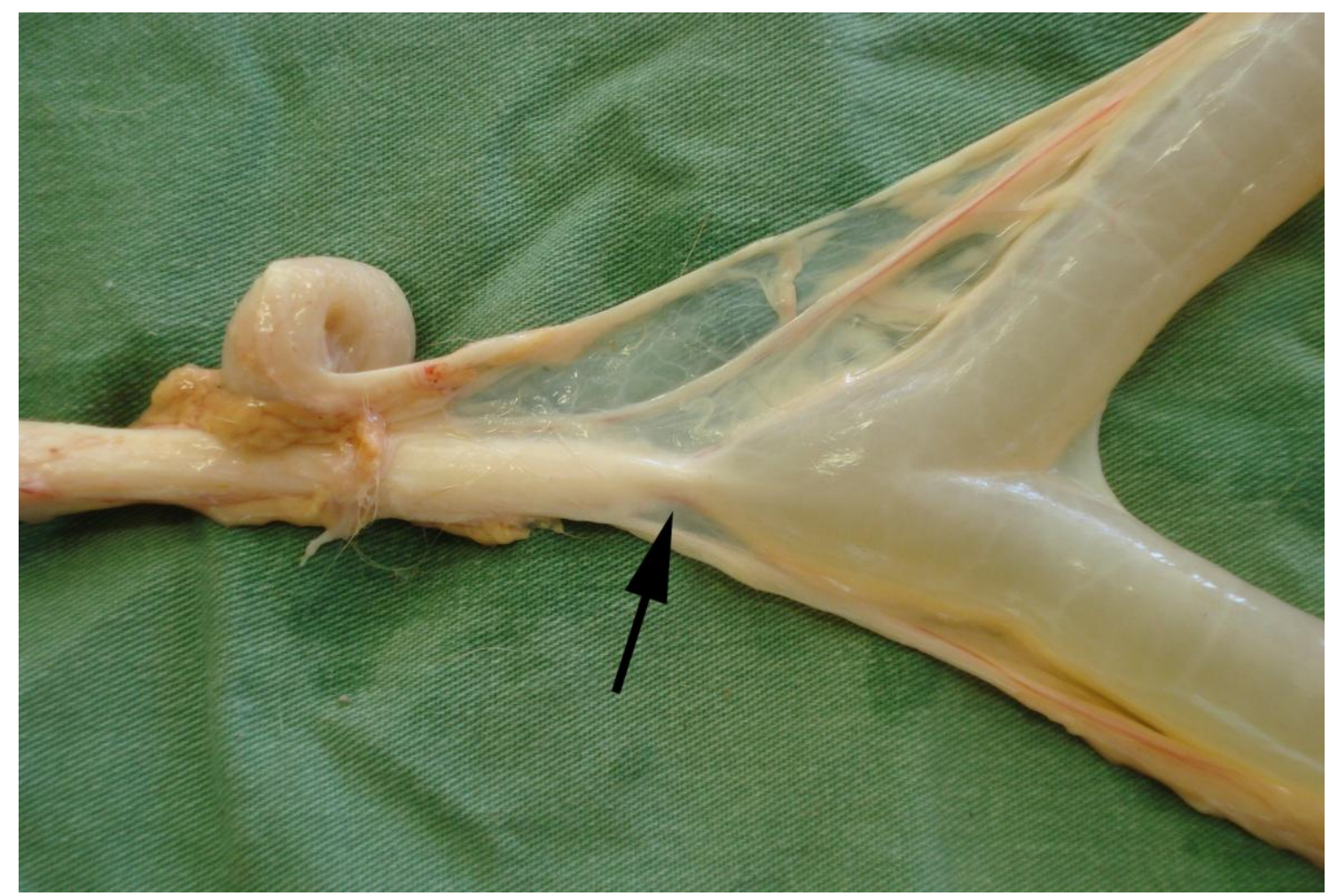

Figura 1. Aplasia segmentar do corpo do útero (seta) com hidrometra nos segmentos craniais. 
Aplasia segmentar de corpo uterino...

Para avaliação histológica, fragmentos dos órgãos genitais foram fixados em formalina tamponada a 10\%, incluídos em parafina, microtomizados e corados por hematoxilina e eosina. Secções transversais da porção cranial da vagina e cérvix mostraram normalidade dessas estruturas. Secções transversais dos cornos uterinos mostraram o perimétrio normal, mas miométrio e endométrio delgados, possuindo o endométrio revestimento epitelial normal, reduzido número de glândulas endometriais com ausência em alguns pontos, epitélio glandular cúbico com núcleo central e ausência de sinais de inflamação, caracterizando hipotrofia endometrial (Fig. 2). Secções longitudinais seriadas do corpo uterino evidenciaram presença de todas as camadas uterinas, entretanto com redução da espessura total do órgão e ausência de lúmen em um segmento, indicando aplasia segmentar do corpo uterino em seu terço mediocaudal, sem comunicação entre as regiões adjacentes (Fig. 3). A região do corpo uterino cranial à aplasia apresentava aspecto histológico semelhante ao encontrado nos cornos, porém a parte caudal apresentava aspecto normal de todas as camadas uterinas. Secções longitudinais dos ovários revelaram a presença bilateral de corpos lúteos em regressão, folículos em várias fases de desenvolvimento e demais estruturas ovarianas normais.

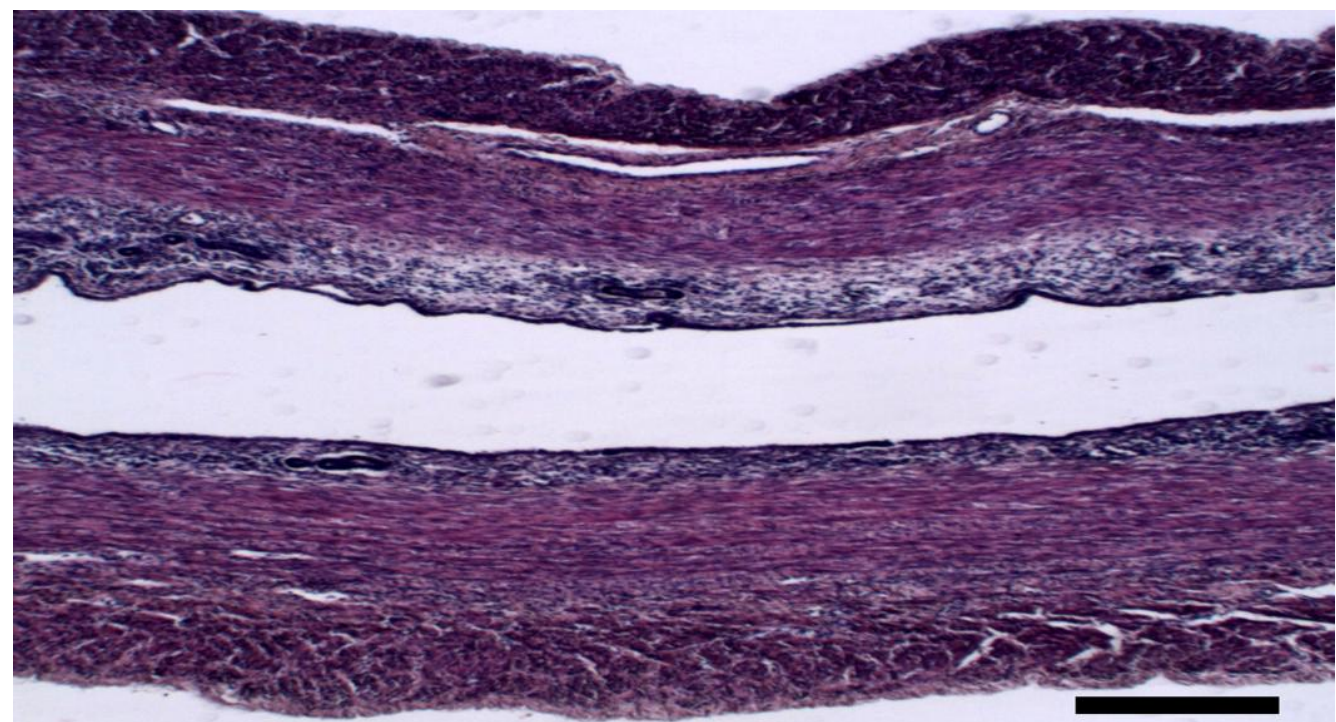

Figura 2. Hipotrofia endometrial acentuada secundária associada à aplasia segmentar. HE. barra=100 $\mu$.

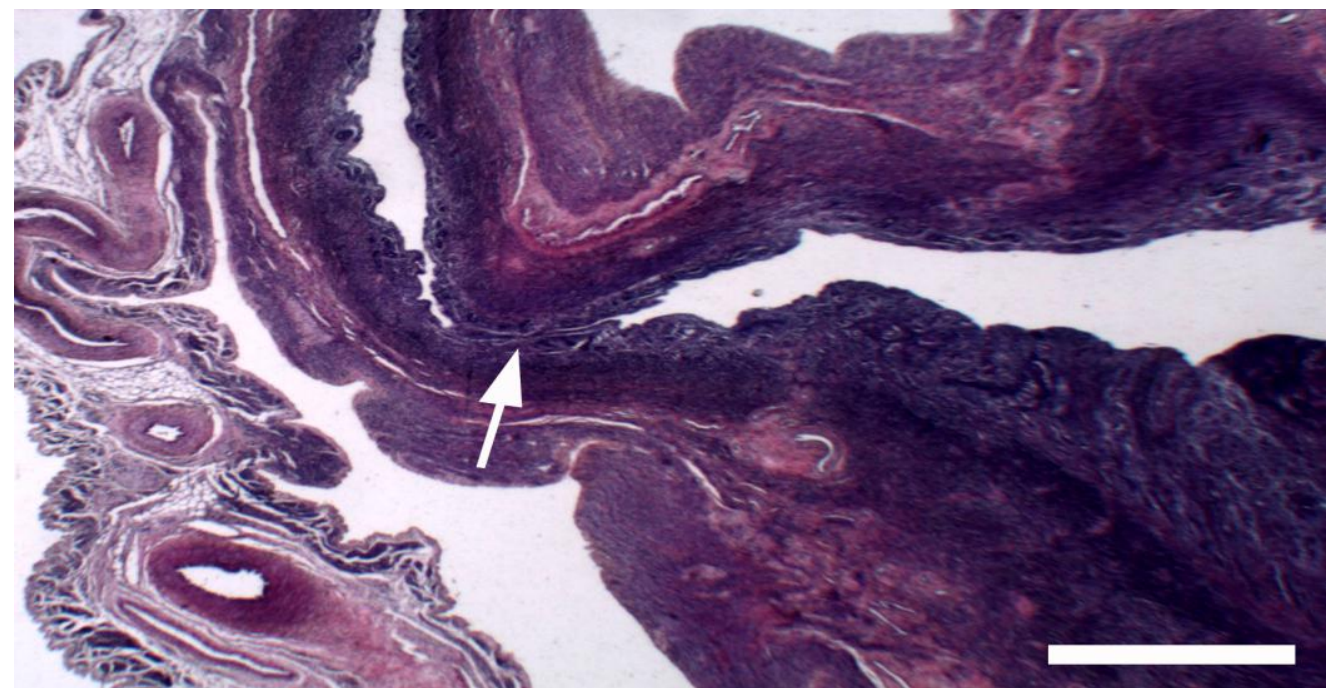

Figura 3. Aplasia segmentar no corpo uterino, com todas as camadas uterinas sem lúmen (seta). HE. barra=500 $\mu$ m. 


\section{DISCUSSÃO}

Segundo a literatura consultada, este é o segundo caso relatado de aplasia segmentar de corpo uterino na cadela, tendo sido anteriormente descrita aplasia completa do corpo uterino com presença de camadas uterinas subdesenvolvidas (Oh et al., 2005). No presente relato, as camadas de tecido uterino subdesenvolvido também estavam presentes e com ausência de luz, mas a extensão da aplasia era de apenas $2 \mathrm{~mm}$ na parte mediocaudal do corpo uterino. Da mesma forma que anteriormente descrita (Oh et al., 2005), os cornos uterinos estavam repletos de fluido, caracterizando hidrometra, neste caso também na parte cranial do corpo uterino. A mucometra e hidrometra são achados comuns associados à aplasia segmentar em outras espécies (Nascimento e Santos, 2003; Schlafer e Miller, 2007).

O complexo hiperplasia endometrial cístico/piometra pode ocorrer associado à aplasia segmentar de cornos uterinos (Prestes et al., 1997; Schulman e Bolton, 1997), interferindo ou não no estado geral dos animais (Schulman e Bolton, 1997). No entanto, neste caso, não houve evidências clínicas, laboratoriais nem morfológicas do complexo. A contaminação bacteriana do fluido uterino acumulado, nos casos de piometra, ocorre por via ascendente, invadindo a luz uterina ao ultrapassar a cérvix durante o estro (Johnston et al., 2001). Mesmo com a possibilidade da passagem de contaminação ascendente pela cérvix, a própria aplasia segmentar impediria a progressão cranial dos agentes microbianos. Nos casos em que ocorreu (Prestes et al., 1997; Schulman e Bolton, 1997), a contaminação microbiana do fluido uterino pode ter se dado por outra via.

A fase de anestro da cadela, identificada inicialmente pela citologia vaginal e dosagem de progesterona sérica, foi confirmada pela presença de corpos lúteos em regressão e folículos ovarianos em várias fases de desenvolvimento nos ovários. Isso corrobora achados anteriores de que a aplasia segmentar do útero não altera a atividade cíclica ovariana na cadela (Johnston et al., 2001), diferente do que ocorre em outras espécies (Nascimento e Santos, 2003; Schlafer e Miller, 2007).
Este relato mostra mais uma vez, como descrito anteriormente (Johnston et al., 2001), que a aplasia segmentar de órgãos genitais da cadela é, na maioria das vezes, achado acidental em laparotomias e exames post morten.

\section{REFERÊNCIAS BIBLIOGRÁFICAS}

JOHNSTON, S.D.; KUSTRITZ, M.V.R.; OLSON, P.N.S. Disorders of the canine uterus and uterine tubes (oviducts). In: JOHNSTON, S.D.; KUSTRITZ, M.V.R.; OLSON, P.N.S. Canine and feline theriogenology. Philadelphia: W.B. Saunders, 2001. p.206-224.

NASCIMENTO, E.F.; SANTOS, R.L. Patologia do sistema genital feminino. In: NASCIMENTO, E.F.; SANTOS, R.L. Patologia da reprodução dos animais domésticos. 2.ed. Rio de Janeiro: Guanabara Koogan, 2003. p.15-89.

OH, K.S.; SON, C.H.; KIM, B.S. et al. Segmental aplasia of uterine body in an adult mixed breed dog. J. Vet. Diag. Invest., v.17, p.490-492, 2005.

ORTEGA-PACHECO, A.; SEGURA-CORREA, J.C.; JIMENEZ-COELLO, M. et al. Reproductive patterns and reproductive pathologies of stray bitches in the tropics. Theriogenology, v.67, p.382-390, 2007.

PRESTES, N.C.; BICUDO, S.D.; LANDIN ALVARENGA, F.C. et al. Aplasia of one uterine horn associated with pyometra in a female dog. Vet. Not., v.3, p.133-134, 1997.

SCHLAFER, D.H.; MILLER, R.B. Female genital system. In: MAXIE, M.G. (Ed). Jubb, Kennedy, and Palmer's Pathology of Domestic Animals. 4.ed. Philadelphia: Elsevier Saunders, 2007. p.429-564.

SCHULMAN, M.L.; BOLTON, L.A. Uterine horn aplasia with complications in two mixedbreed bitches. J. S. Afr. Vet. Assoc., v.68, p.150153, 1997.

WADSWORTH, P.F.; HALL, J.C.; PRENTICE, D.E. Segmental aplasia of the vagina in the beagle bitch. Lab. Anim., v.12, p.165-166, 1978. 